\title{
NCCN Guidelines Update: Breast Cancer
}

\author{
Presented by William Gradishar, MD, and Kilian E. Salerno, MD
}

\begin{abstract}
The updates to management of early invasive breast cancer in 2016 are minor but have important treatment implications for patients. The NCCN Guidelines Panel for Breast Cancer has added endocrine therapy to its recommendations for the neoadjuvant treatment of patients with ER-rich tumors. For women who are premenopausal at diagnosis, the NCCN Guidelines suggest tamoxifen for 5 years, with or without ovarian suppression, or an aromatase inhibitor for 5 years combined with ovarian suppression or ablation. For HER2-positive patients, neoadjuvant pertuzumab is acceptable, and in advanced estrogen receptor-positive disease, palbociclib can be given with endocrine therapy. Hypofractionation is now the preferred approach for whole-breast irradiation after breast-conserving therapy. Regional nodal irradiation should be strongly considered for women with 1 to 3 positive lymph nodes and is indicated for those with 4 or more positive nodes.
\end{abstract}

J Natl Compr Canc Netw 2016;14(5.5):641-644

\section{Important "Tweaks" in Early-Stage Breast Cancer Guidelines}

Updates to the NCCN Clinical Practice Guidelines in Oncology (NCCN Guidelines) for Breast Cancer were relatively minor, according to William Gradishar, MD, the Betsy Bramsen Professor of Breast Oncology at the Feinberg School of Medicine, Northwestern University, and Chair of the NCCN Breast Cancer Guidelines Panel. However, they were called "important tweaks" by Dr. Gradishar and Kilian E. Salerno, MD, Assistant Professor, Radiation Oncology, and Director of Breast Radiation and Soft Tissue/Melanoma Radiation, Roswell Park Cancer Institute, and a member of the NCCN Breast Cancer Panel.

Dr. Gradishar discussed medical management, focusing on endocrine therapy in the neoadjuvant, adjuvant, and metastatic settings; the benefit of ovarian suppression; preoperative HER2-directed therapy; and fertility preservation.

Presented by William Gradishar, MD, Robert H. Lurie Comprehensive Cancer Center of Northwestern University, Chicago, Illinois, and Kilian E. Salerno, MD, Roswell Park Cancer Institute, Buffalo, New York. Drs. Gradishar and Salerno have disclosed that they have no financial interests, arrangements, affiliations, or commercial interests with the manufacturers of any products discussed in this article or their competitors.

Correspondence: William Gradishar, MD, Robert H. Lurie Comprehensive Cancer Center of Northwestern University, 676 N. St. Clair Street, Chicago, IL 60611. E-mail: w-gradishar@northwestern.edu Kilian E. Salerno, MD, Roswell Park Cancer Institute, Elm \& Carlton Streets, Buffalo, NY 14263. E-mail: kilian.salerno@roswellpark.org
Dr. Salerno presented updates in locoregional treatment and emphasized the benefits of hypofractionated whole-breast radiation therapy (RT). She also discussed issues related to regional nodal irradiation.

\section{Neoadjuvant Endocrine Therapy}

For preoperative systemic therapy, the NCCN Guidelines were modified to include the selective use of endocrine therapy. Appropriate candidates are patients with estrogen receptor (ER)-rich tumors (Allred 7-8). Although most of these patients will be postmenopausal, this approach can be considered in premenopausal women with concerns that make them unsuitable for or undesiring of chemotherapy, according to Dr. Gradishar.

Dr. Gradishar referred to ACOSOG Z1031 to show the benefit of neoadjuvant endocrine therapy. After 16 weeks of preoperative exemestane, letrozole, or anastrozole, response or stable disease was observed in $60 \%$, $72 \%$, and $68 \%$ of patients, respectively. ${ }^{1}$

"Adjuvant endocrine therapy is a rational approach," he said. "While this is mainly used in postmenopausal women, you can treat premenopausal women with aromatase inhibitors [AIs] as long as you render them postmenopausal with ovarian suppression or ablation first."

The achievement of pathologic complete response (pCR) is not especially critical with preoperative endocrine therapy, Dr. Gradishar noted. Although pCR is 
Gradishar and Salerno

associated with improved outcomes in patients with ER-negative disease, it does not appreciably impact outcomes in those with ER-positive disease, he said.

\section{Adjuvant Endocrine Therapy Update}

Subtle changes have been made in the recommendations for adjuvant endocrine therapy, reflecting recent trial data. For premenopausal women at diagnosis, the NCCN Guidelines for Breast Cancer suggest tamoxifen for 5 years, with or without ovarian suppression, or an AI for 5 years combined with ovarian suppression or ablation (category 1 ). Women who remain premenopausal can consider tamoxifen for another 5 years (10 total) or no further endocrine therapy. Those who become postmenopausal can consider 5 more years of tamoxifen or switch to an AI for 5 years.

More than half of breast cancer recurrences happen after discontinuation of treatment, ${ }^{2}$ and ovarian suppression or ablation is a way of protecting against these late recurrences. "That maneuver alone has an impact," Dr. Gradishar emphasized. Recently, the SOFT and TEXT trials have confirmed the benefit of ovarian suppression plus endocrine therapy ( \pm chemotherapy). In the joint analysis of these trials (Figure 1), this strategy improved all end points but overall survival. ${ }^{3}$

The findings suggest that ovarian suppression with an AI (exemestane was more effective than tamoxifen) may be appropriate in higher-risk patients; however, musculoskeletal issues and complaints related to estrogen deprivation can be problematic.

"Obviously, these data are in some ways very compelling, but we must individualize our choices," Dr. Gradishar commented.

\section{HER2-Directed Therapy}

Patients with HER2-positive tumors should receive preoperative trastzumumab for 9 weeks or more. The updated NCCN Guidelines accept pertuzumabcontaining regimens as well for patients with $\mathrm{T} 2$ or greater lesions or positive lymph nodes.

In HER2-positive subtypes, pCR is associated with improvements in event-free survival, as shown in a meta-analysis by Cortazar et al. ${ }^{4}$ Whether dual HER2-targeting will ultimately prove the most effective strategy remains to be confirmed, 5,6 but updated preliminary results from NeoSphere showed the greatest improvement in 5-year progression with

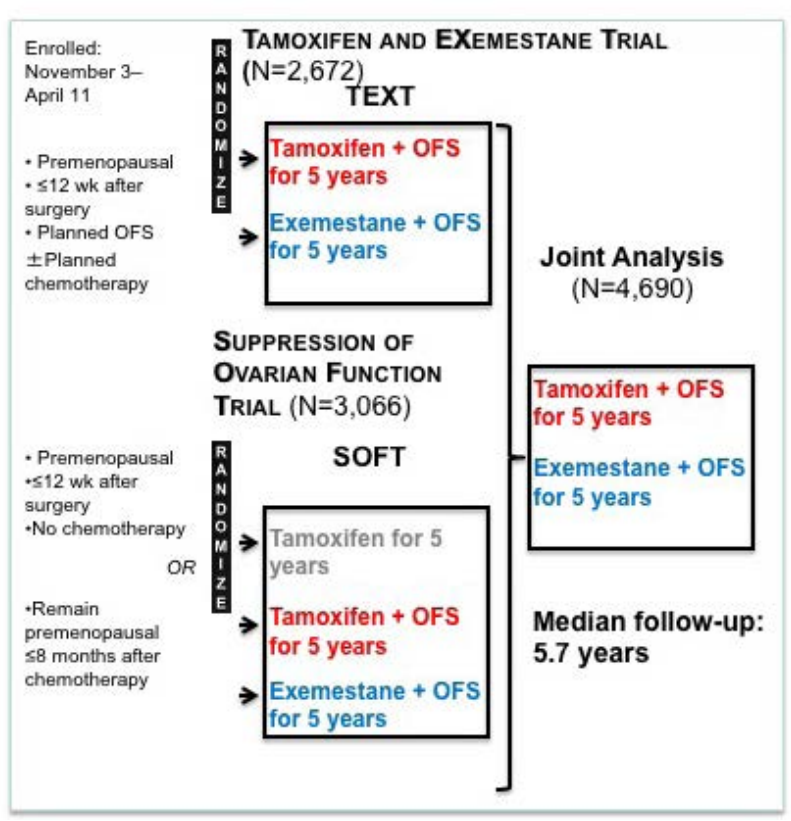

Figure1. Joint analysis of the TEXT and SOFT trials. Abbreviation: OFS, ovarian function suppression.

pertuzumab/trastuzumab/docetaxel. ${ }^{7}$

"Based on the data at this point, we felt it's prudent to offer a pertuzumab-containing regimen to patients," Dr. Gradishar said, adding that the adjuvant APHINITY results will be informative on this issue.

\section{Including Palbociclib}

The NCCN Guidelines for Breast Cancer were modified to reflect the results of the PALOMA trials of palbociclib. This agent joins everolimus as an add-on to endocrine therapy in advanced disease, based on PALOMA-1/TRIO-18. This trial showed an almost doubling in time to progression with letrozole/palbociclib versus letrozole alone in the firstline metastatic setting. ${ }^{8}$ In PALOMA-3, fulvestrant plus palbociclib reduced progression risk by $63 \%$ as second-line treatment. ${ }^{9}$

"Now we have a series of different treatments, and as we go forward we will see even more of this," Dr. Gradishar predicted.

\section{Fertility Preservation}

In another update, the panel recommended that all patients of child-bearing age with early-stage breast cancer see an oncology fertility specialist if they are interested in preserving fertility. A growing number of fertility preservation options have become available. 


\section{Hypofractionation as Preferred RT Strategy}

The NCCN panel now recommends that wholebreast RT be delivered by hypofractionation, a shorter treatment course that uses larger doses per fraction.

"There is no excuse for radiation oncologists not to adopt hypofractionation," said Dr. Salerno.

In short, the guidelines for RT of invasive breast cancer after breast-conserving surgery include the following: RT to the whole breast, with or without a boost; accelerated partial breast irradiation (APBI) in select patients; and omission of RT in select patients, as detailed in an updated "Principles of Radiation Therapy" page (available online, in these guidelines, at NCCN.org).

Whole-breast RT may be delivered using a hypofractionated course of 40 to $42 \mathrm{~Gy}$ in 15 to 16 fractions. This is now the preferred regimen for wholebreast RT after breast-conserving surgery based on clinical trial data from a Canadian trial ${ }^{10}$ and the START-B trial. ${ }^{11}$

Dr. Salerno said that results of hypofractionation are "at least equivalent to or better than" results seen with conventional fractionation in terms of local control, breast cosmesis, and toxicities, and she emphasized it is not to be routinely used in the postmastectomy setting or when treating regional nodes.

For APBI, the panel currently endorses the guidelines issued by the American Society for Radiation Oncology (ASTRO) for determining suitability. ${ }^{12}$ ASTRO is currently drafting a new consensus statement.

Omission of RT altogether is acceptable in select women with lower risk for recurrence. These tend to be patients 70 years old or older with ER-positive, clinically node-negative, T1 tumors who received adjuvant endocrine therapy (category 1 ).

\section{Regional Nodal Irradiation}

The NCCN Guidelines for Breast Cancer include updated language regarding the role of regional nodal irradiation, Dr. Salerno indicated, and detailed the important changes per group:

- 4 or more positive lymph nodes after lumpectomy: RT to whole breast, with or without boost, and regional nodal irradiation (category 1);

- 4 or more positive lymph nodes after mastectomy:
RT to chest wall plus regional nodal irradiation (category1);

- 1 to 3 positive lymph nodes after lumpectomy: RT to whole breast with or without boost (category 1 ); strongly consider regional nodal irradiation based on risk estimate;

- 1 to 3 positive lymph nodes after mastectomy: strongly consider radiation therapy to chest wall plus regional nodal irradiation;

- For regional nodal irradiation, the nodal volumes treated include the supraclavicular area, infraclavicular region, internal mammary nodes, and any part of the axillary bed considered at risk.

The recommendations largely rely on data from the Early Breast Cancer Trialists' Collaborative Group (EBCTCG) meta-analysis ${ }^{13}$ and the 2 most recent trials, MA.2014 and EORTC $22922 .{ }^{15}$ In MA.20 and in EORTC 22922, after 10 years of median follow-up, regional nodal irradiation improved locoregional disease-free survival, distant diseasefree survival, and breast cancer mortality, but not overall survival.

After neoadjuvant therapy, RT is recommended as per maximal stage according to clinical staging presystemic therapy or pathologic staging. For inoperable or locally advanced disease, RT should be delivered to the breast (with or without boost) or chest wall, with regional nodal irradiation.

Treatment of recurrence depends on extent of disease and prior therapies received. These recommendations have been simplified within the updated NCCN Guidelines for Breast Cancer.

\section{References}

1. Olsen JA, Babiera G, Unzeitig GW, et al. ACOSOG Z1031: A randomized phase II trial comparing exemestane, letrozole, and anastrozole in postmenopausal women with clinical stage II/III estrogen receptor-positive breast cancer [abstract]. 2010 Breast Cancer Symposium. Presented October 2, 2010. Abstract 91

2. Early Breast Cancer Trialists' Collaborative Group (EBCTCG). Effects of chemotherapy and hormonal therapy for early breast cancer on recurrence and 15-year survival: an overview of the randomized trials. Lancet 2005;365:1687-1717.

3. Pagani $\mathrm{O}$, Regan MM, Walley $\mathrm{B}$, et al. Randomized comparison of adjuvant aromatase inhibitor (AI) exemestane (E) plus ovarian function suppression (OFS) vs tamoxifen ( $\mathrm{T}$ ) plus OFS in premenopausal women with hormone receptor-positive $(\mathrm{HR}+)$ early breast cancer $(\mathrm{BC})$ : joint analysis of IBCSG TEXT and SOFT trials [abstract]. 2014 ASCO Annual Meeting. Presented May 31, 2014. Abstract LBA1.

4. Cortazar P, Zhang L, Untch M, et al. Pathological complete response and long-term clinical benefit in breast cancer: the CTNeoBC pooled analysis. Lancet 2014;384:164-172.

5. Baselga J, Bradbury I, Eidtmann H, et al. Lapatinib with trastuzumab for HER2-positive early berast cancer (NeoALTTO): a randomized, openlabel, multicenter, phase 3 trial. Lancet 2012;379:633-640. 


\section{Gradishar and Salerno}

6. Gianni L, Pienkowski T, Im YH, et al. Efficacy and safety of neoadjuvant pertuzumab and trastuzumab in women with locally advanced, inflammatory, or early HER2-positive breast cancer (NeoSphere): a randomized multicenter, open-label, phase 2 trial. Lancet Oncol 2012;13:25-32.

7. Gianni L, Pienkowski T, Im Y-H, et al. Five-year analysis of the phase II NeoSphere trial evaluating four cycles of neoadjuvant docetaxel (D) and/ or trastuzumab (T) and/or pertuzumab (P) [abstract]. 2015 ASCO Annual Meeting. Presented June 1, 2015.Abstract 505.

8. Finn RS, Crown JP, Lang ,I et al. The cyclin-dependent kinase $4 / 5$ inhibitor palbociclib in combination with letrozole versus letrozole alone as firstline treatment of oestrogen receptor-positive, HER2-negative, advanced breast cancer (PALOMA-1/TRIO-18): a randomized phase 2 study. Lancet Oncol 2015;16:25-35.

9. Turner NC, Ro J, Andre F, et al. Palbociclib in hormone receptor-positive advanced breast cancer. N Engl J Med 2015;373:209-219.

10. Whelan TJ, Pignol JP, Levine $M N$, et al. Long-term results of hypofractionated radiation therapy for breast cancer. $\mathrm{N}$ Engl J Med 2010;362:513-520.
11. Haviland JS, Owen JR, Dewar JA, et al. The UK Standardisation of Breast Radiotherapy (START) trials of radiotherapy hypofractionation for treatment of early breast cancer: 10-year follow-up results of two randomized controlled trials. Lancet Oncol 2013;14:1086-1094.

12. Smith BD, Arthur DW, Buchholz TA, et al. Accelerated partial breast irradiation consensus statement from the American Society for Radiation Oncology. Int J Radiat Oncol Biol Phys 2009;74:987-1001.

13. Early Breast Cancer Trialists' Collaborative Group. Effect of radiotherapy after mastectomy and axillary surgery on 10-year recurrence and 20-year breast cancer mortality: meta-analysis of individual patient data for 8135 women in 22 randomised trials. Lancet 2014;383:2127-2135.

14. Whelan TJ, Olivotto IA, Parulekar WR, et al. Regional nodal irradiation in early-stage breast cancer. N Engl J Med 2014;373:307-316.

15. Poortmans PM, Collette $S$, Kirkove $C$, et al. Internal mammary and medial supraclavicular irradiation in breast cancer. N Engl J Med 2015;373:317327. 